\section{Caracterización clínica de la incontinencia urinaria y factores asociados en usuarias de la Unidad de la Mujer del Centro de Salud Familiar "Ultraestación" en la ciudad de Chillán, Chile}

\author{
OLGA RINCÓN ARDILA ${ }^{1, \mathrm{a}}$
}

\section{Prevalence and risk factors for urinary incontinence among women consulting in primary care}

Background: It is important to determine the relative importance of urinary incontinence in terms of its prevalence and how it affects the quality of life of women. Aim: To characterize urinary incontinence and factors associated with it in women aged over 30 years. Material and Methods: A survey about urinary incontinence and associated factors was answered by 289 women aged 30 to 81 years, attending a public primary care clinic. Results: The prevalence of urinary incontinence was $62.2 \%$, and it was significantly associated with obesity, diabetes and a history of episiotomy. Conclusions: The high prevalence of urinary incontinence should prompt the implementation of public health measures to prevent and treat it effectively.

(Rev Med Chile 2015; 143: 203-212)

Key words: Prevalence; Urinary incontinence; Women.
${ }^{1}$ Facultad de Ciencias de la Salud y los Alimentos, Universidad del Biobío. Chillán, Chile.

Tesis para optar al título de Magíster en Salud Pública.

${ }^{a}$ Kinesióloga Especialista en

Reeducación pelviperineal.

Recibido el 27 de marzo de 2014, aceptado el 16 de diciembre de 2014.

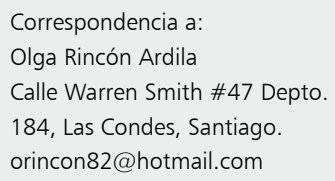

I a incontinencia urinaria (IU), definida por la International Continence Society (ICS) como cualquier pérdida involuntaria de orina, es una enfermedad común, que afecta principalmente a mujeres. Representa un problema de salud pública por su alta prevalencia, según estudios de diversos países y su carga relativa en términos de calidad de vida ${ }^{1-6}$. En la cuarta Consulta Internacional de Continencia, se realizó una revisión de la mejor evidencia disponible y se encontró que la prevalencia de algún grado de IU oscilaba entre 25 y $45 \%$ en mujeres y que entre 7 y $37 \%$ de las mujeres de entre 20 y 39 años reportaban algún episodio de incontinencia de orina diariamente. En las mujeres mayores de 60 años, este porcentaje varió entre 9 y $39 \%{ }^{7}$. En Chile, Salazar y colabo- radores determinaron una prevalencia de IU de $62,2 \%$ en una población cerrada ${ }^{8}$ y Hardwardt y colaboradores determinaron una prevalencia de $77 \%$ en mujeres de Isla de Pascua ${ }^{9}$. A pesar de que la IU no representa una amenaza para la vida, tiene una influencia negativa sobre la condición física, psicológica y social de las mujeres y está asociada con una importante disminución en la calidad de vida e importantes costos directos e indirectos en salud $^{10-14}$.

La mayoría de los estudios epidemiológicos sobre prevalencia de IU y factores asociados han sido realizados en Europa y Estados Unidos de Norteamérica. No existen estudios en Chile que demuestren la importancia de esta alteración en las usuarias de establecimientos de atención 
primaria. Igualmente, hay abundante evidencia internacional sobre factores de riesgo que se asocian al desarrollo o presencia de $\mathrm{IU}^{3,5,15,16,17}$, entre los que se encuentran la edad avanzada $3,5,6,16$, el aumento del índice de masa corporal ${ }^{18,19,20,21}$, la diabetes mellitus ${ }^{22}$, los embarazos y partos ${ }^{23,24,25,26}$, la historia de histerectomía ${ }^{26,28,29,30}$, y el inicio de la menopausia ${ }^{26,27}$. El presente estudio busca caracterizar la IU y los factores asociados a ésta en las mujeres que consultan en una unidad de la mujer en atención primaria, para determinar su peso relativo y así poder destinar recursos para su prevención y tratamiento.

\section{Material y Método}

Se realizó un estudio de corte transversal correlacional, aplicando una encuesta a $289 \mathrm{mu}-$ jeres mayores de 30 años inscritas en el CESFAM Ultraestación de Chillán, que asistían a control en la unidad de la mujer en el período entre octubre de 2012 y enero de 2013. Fueron excluidas las mujeres embarazadas y aquellas que acudieran a consultar por sintomatología de vía urinaria baja. La aceptación y respuesta del cuestionario fue voluntaria y anónima, posterior a la explicación de los objetivos del estudio. El cuestionario contenía preguntas cerradas y categorizadas sobre datos demográficos, presencia y severidad de IU, historia médica y quirúrgica, historia ginecobstétrica, y sobre consultas previas por IU. El cuestionario fue validado a través de la opinión de expertos y la realización de una prueba piloto a 32 mujeres de similares características a las de la muestra. Se definió la IU como pérdidas involuntarias de orina en el último mes y la severidad se determinó a través del índice de Sandvik ${ }^{31}$ (Tabla 1).

Los análisis estadísticos se realizaron con ayuda del software estadístico SPSS versión 20. Para el cálculo de razón de prevalencias en variables independientes con más de dos categorías se usó Stata 9.0. Se realizaron estimaciones de prevalencia generales, y estimaciones de la distribución de severidad y tipo de IU. Las posibles relaciones bivariadas entre factores de riesgo potenciales y la prevalencia de IU se midieron mediante razones de prevalencia con sus intervalos de confianza (95\%) y test de chi cuadrado y de Fisher. Se realizó una correlación entre la severidad y los diferentes factores asociados para identificar un aumento en la probabilidad de presentar una mayor severidad al estar expuesto a cada factor. También se realizó una correlación entre el tipo de IU y los diferentes factores asociados para identificar posibles asociaciones para cada tipo de IU. Un valor $\mathrm{p}<0,05$ se consideró estadísticamente significativo.

\section{Resultados}

Se encuestaron 289 mujeres entre 30 y 81 años en un período de 3 meses, entre octubre de 2012 y enero de 2013. La Tabla 2 muestra las características generales de las participantes del estudio, quienes tenían un promedio de 47 años. En cuanto al estado nutricional, la mayoría de las participantes presentaban sobrepeso y obesidad.

\section{Tabla 1. Índice de Sandvik}

(I) ¿Con qué frecuencia experimenta pérdidas de orina?
1. Menos de una vez al mes
2. Una o más veces al mes
3. Una o más veces a la semana
4. Todos los días y/o noches
(II) ¿Qué tanta orina pierde en cada episodio?
1. Gotas
2. Chorro
3. Más
Índice de Severidad (1) x(2)
1-2 = Leve
3-6 = Moderada
$8-9=$ Severa
$12=$ Muy Severa

\section{Tabla 2. Características generales de las participantes del estudio $(n=289)$}

\begin{tabular}{|lrc|}
\hline Antecedentes & $\overline{\mathbf{x}} \pm \mathbf{D E}$ & Mínimo-Máximo \\
\hline Edad (años) & $47,66 \pm 11,31$ & $30-81$ \\
IMC & $29,12 \pm 5,92$ & $17,5-67,3$ \\
\hline Estado Nutricional $^{\dagger}$ & & \\
$\quad$ Enflaquecido & $4(1,4)$ & \\
$\quad$ Normal & $61(21,1)$ \\
$\quad$ Sobrepeso & $116(40,1)$ \\
$\quad$ Obeso $^{\text {Diabetes }}{ }^{\dagger}$ & $108(37,3)$ \\
Dianstipación $^{\dagger}$ & $30(10,4)$ \\
Consti $^{\dagger}$ (\%). & $103(35,6)$ \\
\hline
\end{tabular}


Tabla 3. Características gineco-obstétricas de las participantes del estudio $(\mathbf{n}=\mathbf{2 8 9})$

\begin{tabular}{|lcc|}
\hline Características gineco-obstétricas & $\mathbf{n}(\mathbf{\%})$ & Mínimo-Máximo \\
n embarazos $^{\dagger}$ & $2,52 \pm 1,69$ & $0-12$ \\
Mecanismo de parto & $17(5,9)$ \\
$\quad$ Ninguno & $213(73,7)$ \\
$\quad$ Al menos uno vaginal & $59(20,4)$ \\
Sólo cesárea & $3.533,6 \pm 564,5$ \\
Peso de recién nacido $(\mathrm{g})^{\dagger}$ & $173(81,2)$ \\
Episiotomía $(\mathrm{n}=213)$ & $24(11,3)$ \\
Parto instrumental $(\mathrm{n}=213)$ & $112(38,8)$ \\
Menopausia & $3(2,7)$ \\
Reemplazo hormonal $(\mathrm{n}=112)$ & $21 \quad(7,3)$ \\
Histerectomía & $1.550-5.200$ \\
\hline
\end{tabular}

${ }^{+} \overline{\mathbf{X}} \pm(\mathrm{DE})$.

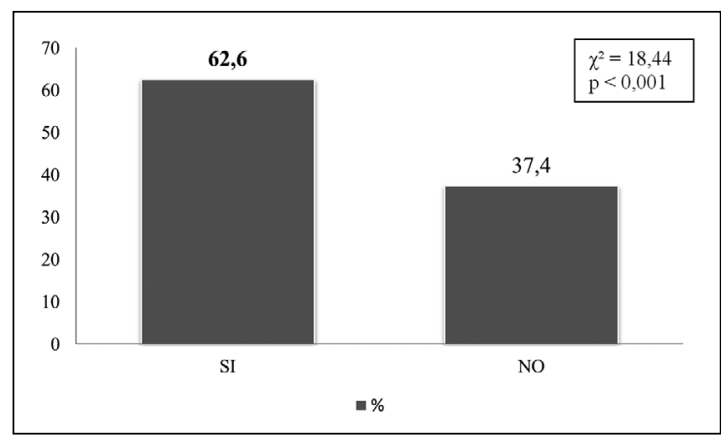

Figura 1. Prevalencia de incontinencia urinaria de las participantes del estudio $(n=289)$.

La Tabla 3 presenta las características ginecobstétricas de las participantes. La mayoría de ellas había experimentado al menos un parto vaginal, con un alto porcentaje de episiotomía y un bajo porcentaje de partos instrumentales.

La prevalencia de incontinencia urinaria en el grupo estudiado fue de $62,6 \%$ (Figura 1). La mayoría de mujeres con IU presentaron una severidad leve y moderada de ésta, mientras que sólo $7,8 \%$ presentó IU muy severa (Tabla 4). La incontinencia más frecuente en este grupo fue la de esfuerzo (IUE), seguida por la incontinencia mixta (IUM) (Tabla 5).

La relación entre las variables propuestas como posibles factores de riesgo y la presencia de IU se muestra en la Tabla 6. Respecto a la edad no se demostró diferencia significativa entre las mujeres
Tabla 4. Severidad de la incontinencia urinaria de las participantes del estudio $(n=181)$

\begin{tabular}{|lcc|}
\hline Grado de incontinencia urinaria $^{\dagger}$ & $\mathbf{n}$ & $\mathbf{\%}$ \\
\hline Leve & 68 & 37,6 \\
Moderada & 76 & 42,2 \\
Severa & 23 & 12,8 \\
Muy severa & 14 & 7,8 \\
\hline
\end{tabular}

tEscala de Sandvik.

Tabla 5. Clasificación por tipo de incontinencia urinaria de las participantes del estudio ( $n=181)$

\begin{tabular}{|lrr|}
\hline Tipo de incontinencia urinaria & n & \multicolumn{1}{c|}{$\%$} \\
\hline Incontinencia de esfuerzo & 111 & 61,3 \\
\hline Urgeincontinencia & 18 & 9,9 \\
\hline Incontinencia mixta & 52 & 28,7 \\
\hline
\end{tabular}

con IU y las que no la presentaban, en todos los grupos de edad. Se encontró que $60,3 \%$ de las mujeres con sobrepeso experimentan IU, teniendo 1,12 veces más probabilidad de presentar IU que aquellas con estado nutricional normal. El 72,2\% de las mujeres obesas reportaron IU, lo que les confiere 1,33 veces más probabilidad de presentar dicha patología, siendo la diferencia estadísticamente significativa para este grupo de mujeres. A 
pesar de no haber diferencia estadísticamente significativa, aquellas mujeres que tuvieron al menos un embarazo tuvieron una mayor probabilidad de experimentar IU al compararlas con las mujeres nulíparas. Las mujeres que experimentan partos vaginales presentan 1,38 veces más probabilidad de desarrollar IU al compararlas con mujeres que no habían experimentado partos. De las 24 mujeres que reportaron haber sido sometidas a parto instrumental, $15(62,5 \%)$ reportaron IU, sin

Tabla 6. Presencia de incontinencia urinaria y factores asociados $(n=289)$

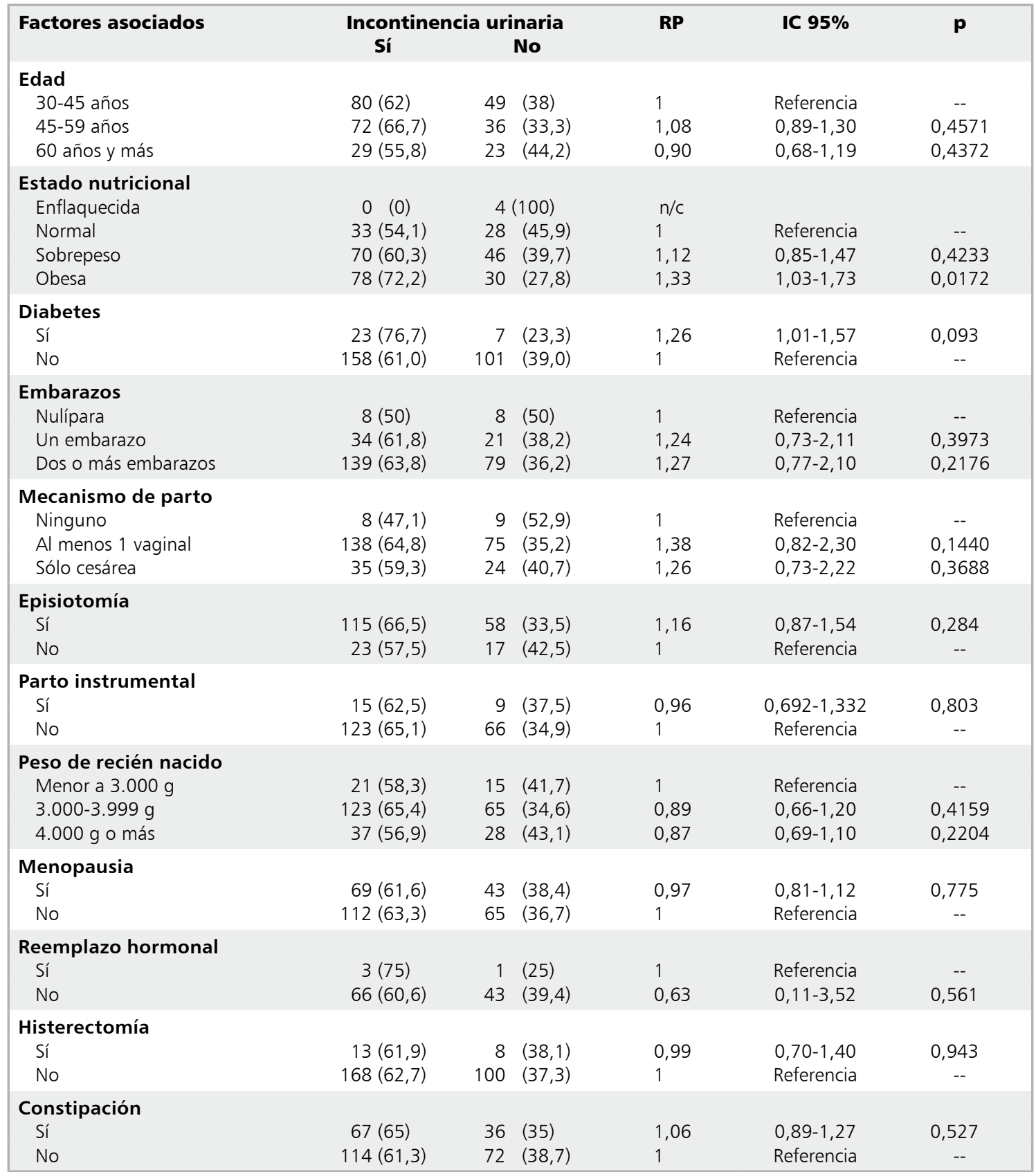

$\mathrm{n}(\%)$. RP = razón de prevalencia, IC 95\% = Intervalo de confianza; prueba de independencia $\left(\chi^{2}\right)$. 
embargo no hubo diferencias significativas con el grupo que no fue sometido a este procedimiento.

La relación entre las variables propuestas como posibles factores de riesgo y la severidad de incontinencia urinaria se muestra en la Tabla 7.
En mayores de 45 años aumentó 1,84 veces la probabilidad de presentar IU de mayor severidad, aunque no es estadísticamente significativo. Respecto a los factores obstétricos, ni la cantidad, ni el mecanismo de parto, ni la exposición a

Tabla 7. Severidad de la incontinencia urinaria y factores asociados $(n=180)$

\begin{tabular}{|c|c|c|c|c|c|}
\hline Factores asociados & \multicolumn{2}{|c|}{ Incontinencia urinaria } & $\mathbf{R P}$ & IC $95 \%$ & $\mathbf{p}$ \\
\hline \multicolumn{6}{|l|}{ Edad } \\
\hline 30-45 años & $69(86,2)$ & $11(13,8)$ & 1 & Referencia & -- \\
\hline 45-59 años & $53(74,6)$ & $18(25,4)$ & 1,84 & $0,94-3,63$ & 0,0709 \\
\hline 60 años y más & $21(72,4)$ & $8(27,6)$ & 1,84 & $0,94-3,63$ & 0,0925 \\
\hline \multicolumn{6}{|l|}{ Estado nutricional } \\
\hline Normal & $26(78,8)$ & $7(21,2)$ & 1 & Referencia & -- \\
\hline Sobrepeso & $58(84,1)$ & $11(15,9)$ & 0,75 & $0,32-1,76$ & 0,5136 \\
\hline Obesa & $59(75,6)$ & $19(24,4)$ & 1,15 & $0,53-2,47$ & 0,7205 \\
\hline \multicolumn{6}{|l|}{ Diabetes } \\
\hline Sí & $18(69,6)$ & $7(30,4)$ & 1,60 & $0,79-3,20$ & 0,2093 \\
\hline No & $127(80,9)$ & $30(19,1)$ & 1 & Referencia & -- \\
\hline \multicolumn{6}{|l|}{ Embarazos } \\
\hline Nulípara & $6(75,0)$ & $2(25,0)$ & 1 & Referencia & -- \\
\hline Sólo uno & $27(81,8)$ & $6(18,2)$ & 0,73 & $0,18-2,95$ & 0,642 \\
\hline Dos o más & $110(79,1)$ & $29(20,9)$ & 0,83 & $0,24-2,89$ & 0,676 \\
\hline \multicolumn{6}{|l|}{ Mecanismo de parto } \\
\hline Ninguno & $6(75,0)$ & $2(25,0)$ & 1 & Referencia & -- \\
\hline Al menos 1 vaginal & $106(77,4)$ & $31(22,6)$ & 0,91 & $0,26-3,13$ & 1,000 \\
\hline Sólo cesárea & $31(88,6)$ & $4(11,4)$ & 0,45 & $0,10-2,08$ & 0,308 \\
\hline \multicolumn{6}{|l|}{ Episiotomía } \\
\hline Sí & $90(78,9)$ & $24(21,1)$ & 0,69 & $0,34-1,41$ & 0,3266 \\
\hline No & $16(69,6)$ & $7(30,4)$ & 1 & Referencia & -- \\
\hline \multicolumn{6}{|l|}{ Parto instrumental } \\
\hline Sí & $10(66,7)$ & $5(33,3)$ & 1,56 & $0,71-3,46$ & 0,2937 \\
\hline No & $96(78,7)$ & $26(21,3)$ & 1 & Referencia & -- \\
\hline \multicolumn{6}{|l|}{ Peso de recién nacido } \\
\hline Menor a $3.000 \mathrm{~g}$ & $16(76,2)$ & $5(23,8)$ & 1 & Referencia & -- \\
\hline $3.000-3.999 \mathrm{~g}$ & $97(79,5)$ & $25(20,5)$ & 0,86 & $0,37-2,00$ & 0,773 \\
\hline 4.000 g o más & $30(81,1)$ & $7(18,9)$ & 0,79 & $0,29-2,20$ & 0,741 \\
\hline \multicolumn{6}{|l|}{ Menopausia } \\
\hline Sí & $51(75,0)$ & $17(25,0)$ & 1,4 & $0,79-2,50$ & 0,2502 \\
\hline No & $92(82,1)$ & $20(17,9)$ & 1 & Referencia & -- \\
\hline \multicolumn{6}{|l|}{ Reemplazo hormonal } \\
\hline Sí & $3(100)$ & $0(0,0)$ & 1 & Referencia & -- \\
\hline No & $48(73,8)$ & $17(26,2)$ & & $\mathrm{N} / \mathrm{C}$ & \\
\hline \multicolumn{6}{|l|}{ Histerectomía } \\
\hline Sí & $10(76,9)$ & $3(23,1)$ & 1,13 & $0,40-3,20$ & 0,8153 \\
\hline No & $133(79,6)$ & $34(20,4)$ & 1 & Referencia & - \\
\hline \multicolumn{6}{|l|}{ Constipación } \\
\hline Sí & $47(71,2)$ & $19(28,8)$ & 1,82 & $1,03-3,22$ & 0,0376 \\
\hline No & $96(84,2)$ & $18(15,8)$ & 1 & Referencia & -- \\
\hline
\end{tabular}

$\mathrm{n}(\%) . \mathrm{RP}=$ razón de prevalencia, IC 95\% = Intervalo de confianza; prueba de independencia $\left(\chi^{2}\right)$ 
Caracterización clínica de la incontinencia urinaria en mujeres - O. Rincón Ardila

episiotomía, ni el peso de recién nacido aumentaron la probabilidad de presentar IU de mayor severidad. Sólo el parto instrumental mostró una probabilidad 1,56 veces mayor de presentar IU más severa. La constipación aumentó 1,82 veces la probabilidad de desarrollar IU de tipo más severo, siendo la asociación estadísticamente significativa.

La relación entre las variables propuestas como posibles factores de riesgo y el tipo de incontinencia urinaria se muestra en la Tabla 8 . Aunque sin

Tabla 8. Tipo de Incontinencia urinaria y factores asociados $(n=289)$

\begin{tabular}{|c|c|c|c|c|c|c|c|}
\hline Factores asociados & $\begin{array}{r}{ }^{T} \\
\text { Esfuerzo }\end{array}$ & $\begin{array}{l}\text { o de incon } \\
\text { Urgencia }\end{array}$ & $\begin{array}{c}\text { nencia uri } \\
\text { Mixta }\end{array}$ & $\begin{array}{l}\text { lario } \\
\text { Continente }\end{array}$ & $\begin{array}{c}\text { RA } \\
\text { esfuerzo }\end{array}$ & $\begin{array}{c}\mathbf{R A} \\
\text { urgencia }\end{array}$ & $\begin{array}{c}\text { RA } \\
\text { mixta }\end{array}$ \\
\hline $\begin{array}{l}\text { Edad } \\
\text { 30-45 años } \\
45-59 \text { años } \\
60 \text { años y más }\end{array}$ & $\begin{array}{l}52(40,3) \\
49(45,4) \\
10(19,2)\end{array}$ & $\begin{array}{lr}8 & (6,2) \\
4 & (3,7) \\
6 & (11,5)\end{array}$ & $\begin{array}{l}20(15,5) \\
19(17,6) \\
13(25)\end{array}$ & $\begin{array}{l}49(38) \\
36(33,3) \\
23(44,2)\end{array}$ & $\begin{array}{l}1,00 \\
1,12 \\
0,60^{*}\end{array}$ & $\begin{array}{l}1,00 \\
0,71 \\
1,47\end{array}$ & $\begin{array}{l}1,00 \\
1,20 \\
1,25\end{array}$ \\
\hline $\begin{array}{l}\text { Estado nutricional } \\
\text { Normal } \\
\text { Sobrepeso } \\
\text { Obesa }\end{array}$ & $\begin{array}{l}15(24,6) \\
49(42,2) \\
47(43,5)\end{array}$ & $\begin{array}{lr}7 & (11,5) \\
7 & (6,0) \\
4 & (3,7)\end{array}$ & $\begin{array}{l}11(18) \\
14(12,1) \\
27(25)\end{array}$ & $\begin{array}{l}28(45,9) \\
46(39,7) \\
30(27,8)\end{array}$ & $\begin{array}{l}1,00 \\
1,48 \\
1,75^{*}\end{array}$ & $\begin{array}{l}1,00 \\
0,66 \\
0,59\end{array}$ & $\begin{array}{l}1,00 \\
0,83 \\
1,68\end{array}$ \\
\hline $\begin{array}{l}\text { Diabetes } \\
\text { Sí } \\
\text { No }\end{array}$ & $\begin{array}{c}10(33,3) \\
101(39)\end{array}$ & $\begin{aligned} 2 & (6,7) \\
16 & (6,2)\end{aligned}$ & $\begin{array}{l}11(36,7) \\
41(15,8)\end{array}$ & $\begin{aligned} 7 & (23,3) \\
101 & (39)\end{aligned}$ & $\begin{array}{l}1,18 \\
1,00\end{array}$ & $\begin{array}{l}1,63 \\
1,00\end{array}$ & $\begin{array}{c}2,12^{*} \\
1,00\end{array}$ \\
\hline $\begin{array}{l}\text { Embarazos } \\
\text { Nulípara } \\
\text { Sólo uno } \\
\text { Dos o más }\end{array}$ & $\begin{array}{c}4(25) \\
23(41,8) \\
84(38,5)\end{array}$ & $\begin{array}{rr}1 & (6,2) \\
4 & (7,3) \\
13 & (6,0)\end{array}$ & $\begin{array}{r}3(18,8) \\
7(12,7) \\
42(19,3)\end{array}$ & $\begin{array}{c}8(50) \\
21(38,2) \\
79(36,2)\end{array}$ & $\begin{array}{l}1,00 \\
1,57 \\
1,55\end{array}$ & $\begin{array}{l}1,00 \\
1,44 \\
1,27\end{array}$ & $\begin{array}{l}1,00 \\
0, .92 \\
1,27\end{array}$ \\
\hline $\begin{array}{l}\text { Mecanismo de parto } \\
\text { Ninguno } \\
\text { Al menos } 1 \text { vaginal } \\
\text { Sólo cesárea }\end{array}$ & $\begin{array}{r}4(23,5) \\
85(39,9) \\
22(37,3)\end{array}$ & $\begin{aligned} 1 & (5,9) \\
12 & (5,6) \\
5 & (8,5)\end{aligned}$ & $\begin{array}{r}3(17,6) \\
41(19,2) \\
8(13,6)\end{array}$ & $\begin{array}{r}9(52,9) \\
75(35,2) \\
24(40,7)\end{array}$ & $\begin{array}{l}1,00 \\
1,73 \\
1,55\end{array}$ & $\begin{array}{l}1,00 \\
1,38 \\
1,72\end{array}$ & $\begin{array}{l}1,00 \\
1,41 \\
1,00\end{array}$ \\
\hline $\begin{array}{l}\text { Episiotomía } \\
\text { Sí } \\
\text { No }\end{array}$ & $\begin{array}{c}77(44,5) \\
8(20)\end{array}$ & $\begin{array}{l}8(4,6) \\
4(10)\end{array}$ & $\begin{array}{l}30(17,3) \\
11(27,5)\end{array}$ & $\begin{array}{l}58(33,5) \\
17(42,5)\end{array}$ & $\begin{array}{l}1,78^{*} \\
1,00\end{array}$ & $\begin{array}{l}0,64 \\
1,00\end{array}$ & $\begin{array}{l}0,87 \\
1,00\end{array}$ \\
\hline $\begin{array}{l}\text { Parto instrumental } \\
\text { Sí } \\
\text { No }\end{array}$ & $\begin{array}{r}7(29,2) \\
78(41,3)\end{array}$ & $\begin{aligned} 1 & (4,2) \\
11 & (5,8)\end{aligned}$ & $\begin{array}{l}7(29,2) \\
34(18)\end{array}$ & $\begin{array}{r}9(37,5) \\
66(34,9)\end{array}$ & $\begin{array}{l}0,81 \\
1,00\end{array}$ & $\begin{array}{l}0,70 \\
1,00\end{array}$ & $\begin{array}{l}1,28 \\
1,00\end{array}$ \\
\hline $\begin{array}{l}\text { Peso de recién nacido } \\
<3.000 \mathrm{~g} \\
3.000-3.999 \mathrm{~g} \\
4.000 \mathrm{~g} \text { o más }\end{array}$ & $\begin{array}{l}10(27,8) \\
82(43,6) \\
19(29,2)\end{array}$ & 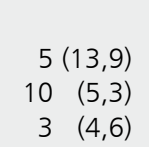 & $\begin{array}{r}6(16,7) \\
31(16,5) \\
15(23,1)\end{array}$ & $\begin{array}{l}15(41,7) \\
65(34,6) \\
28(43,1)\end{array}$ & $\begin{array}{l}0,72 \\
1,00 \\
0,72\end{array}$ & $\begin{array}{l}1,88 \\
1,00 \\
0,73\end{array}$ & $\begin{array}{l}0,88 \\
1,00 \\
1,10\end{array}$ \\
\hline $\begin{array}{l}\text { Menopausia } \\
\text { Sí } \\
\text { No }\end{array}$ & $\begin{array}{l}35(31,2) \\
76(42,9)\end{array}$ & 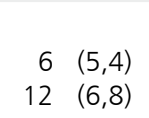 & $\begin{array}{l}28(25) \\
24(13,6)\end{array}$ & $\begin{array}{l}43(38,4) \\
65(36,7)\end{array}$ & $\begin{array}{l}0,83 \\
1,00\end{array}$ & $\begin{array}{l}0,78 \\
1,00\end{array}$ & $\begin{array}{l}1,46 \\
1,00\end{array}$ \\
\hline $\begin{array}{l}\text { Reemplazo hormonal } \\
\text { Sí } \\
\text { No }\end{array}$ & $\begin{array}{c}2(50) \\
33(30,3)\end{array}$ & $\begin{array}{l}1(25) \\
5 \quad(4,6)\end{array}$ & $\begin{array}{cc}0(0) \\
28(25,7)\end{array}$ & $\begin{array}{c}1(25) \\
43(39,4)\end{array}$ & $\begin{array}{l}1,00 \\
1,53\end{array}$ & $\begin{array}{l}1,00 \\
4,8\end{array}$ & $\begin{array}{c}1,00 \\
n / c\end{array}$ \\
\hline $\begin{array}{l}\text { Histerectomía } \\
\text { Sí } \\
\text { No }\end{array}$ & $\begin{array}{r}8(38,1) \\
103(38,4)\end{array}$ & $\begin{array}{rr}1 & (4,8) \\
17 & (6,3)\end{array}$ & $\begin{array}{c}4(19) \\
48(17,9)\end{array}$ & $\begin{array}{r}8(38,1) \\
100(37,3)\end{array}$ & $\begin{array}{l}0,99 \\
1,00\end{array}$ & $\begin{array}{l}0,76 \\
1,00\end{array}$ & $\begin{array}{l}1,03 \\
1,00\end{array}$ \\
\hline $\begin{array}{l}\text { Constipación } \\
\text { Sí } \\
\text { No }\end{array}$ & $\begin{array}{l}36(35) \\
75(40,3)\end{array}$ & $\begin{aligned} 6 & (5,8) \\
12 & (6,5)\end{aligned}$ & $\begin{array}{l}25(24,3) \\
27(14,5)\end{array}$ & $\begin{array}{l}36(35) \\
72(38,7)\end{array}$ & $\begin{array}{l}0,98 \\
1,00\end{array}$ & $\begin{array}{l}1,00 \\
1,00\end{array}$ & $\begin{array}{l}1,50 \\
1,00\end{array}$ \\
\hline
\end{tabular}

$n(\%) . R A=$ Razón de prevalencia. Prueba de Independencia: ${ }^{*} p<0,05^{* *} p<0,01{ }^{* * *} p<0,001$. 
Tabla 9. Consultas por IU $(n=289)$

\begin{tabular}{|lcc|}
\hline & $\mathbf{n}$ & $\mathbf{\%}$ \\
\hline Total de consultas por IU & 31 & 10,7 \\
Profesional $(\mathrm{n}=31)$ & & \\
Matrona & 21 & 67,7 \\
Médico APS & 3 & 9,7 \\
Médico general particular & 2 & 6,4 \\
Ginecólogo & 2 & 6,4 \\
Urólogo & 3 & 9,7 \\
\hline
\end{tabular}

diferencia significativa, las mujeres de 60 años y más presentaron una mayor probabilidad de presentar IU e IUM. Este grupo etario presentó una menor probabilidad de presentar IUE, siendo esta asociación estadísticamente significativa. La obesidad aumentó 1,75 veces la probabilidad de presentar IUE, siendo esta asociación estadísticamente significativa. La diabetes mellitus aumentó la probabilidad de desarrollar todos los tipos de IU, siendo 2,12 veces mayor la probabilidad de presentar IUM en las mujeres diabéticas, asociación que fue estadísticamente significativa. Las mujeres a las que se les realizó episiotomía tuvieron una probabilidad 1,78 veces mayor de presentar IUE, siendo este hallazgo estadísticamente significativo.

La Tabla 9 muestra la caracterización de las consultas por IU. La tasa de consulta por esta patología fue baja, con sólo $10,7 \%$ de las mujeres con IU consultando a algún profesional de la salud. La mayoría consultó con la matrona de atención primaria durante algún control o consulta. Sólo una minoría de mujeres consultó con algún otro profesional médico.

\section{Discusión}

La prevalencia total de IU en el presente estudio concuerda con la reportada en la cuarta Consulta Internacional sobre Incontinencia, que corresponde a una revisión de 36 estudios de prevalencia, y que encontró que la prevalencia de incontinencia en mujeres a nivel global variaba entre $7 \%$ y $63 \%$, estando la mayoría de los resultados entre 25 y $45 \%$. También concuerda con el estudio chileno de Salazar et al, quienes reportaron una prevalencia de $62,2 \%$ en mujeres de similares características a las encuestadas en el presente estudio ${ }^{8}$.
A pesar de que la evidencia, tanto de este estudio como la reportada por otros autores, demuestra sin lugar a dudas que la IU es un problema común, establecer prevalencias precisas que sirvan para apoyar acciones de salud pública ha sido complejo. Una razón de esto es la dificultad para comparar los estudios disponibles, ya sea por presentar diferencias metodológicas, en las muestras seleccionadas, en los instrumentos utilizados y especialmente en la definición de IU. En este estudio la presencia de IU fue establecida según la definición de la ICS, a través del auto-reporte de las mujeres encuestadas. La mayoría de los estudios epidemiológicos utilizan el auto-reporte de IU de las participantes. Varios autores han comparado el auto-reporte con la evaluación clínica de IU encontrando que existe una alta correlación ${ }^{31}$. Desde el punto de vista de salud pública el auto-reporte presenta la ventaja de reflejar la experiencia de la mujer y permite una mejor caracterización de la incontinencia por su frecuencia y cantidad de las pérdidas urinarias. La desventaja del instrumento utilizado en este estudio, principalmente en relación a los factores asociados, es el posible sesgo de información y memoria.

En este estudio se encontró una prevalencia de IU mayor en las mujeres entre 46 y 59 años que en aquellas de menor edad, pero la prevalencia en las mujeres de 60 o más años fue un poco inferior. Esto puede relacionarse con varios factores, uno el aspecto cultural, ya que por tratarse de autoreporte, la vergüenza o la idea de que la IU es un aspecto "normal" o inevitable del envejecimiento puede haber influido en la respuesta de las mujeres mayores al cuestionario, en segundo lugar este hecho puede relacionarse con el diseño transversal del estudio, y el hecho de que la muestra de mujeres de este último grupo etario fue reducida, por lo que no puede descartarse sesgo de selección. Otros estudios han reportado una prevalencia reducida de IU en el grupo de mujeres entre 67-79 años (6,31-33), las razones de que esto ocurra no son claras, pero se ha postulado que puede estar asociado con factores hormonales o con una reducción en el nivel de actividad física a medida que las mujeres envejecen, lo cual explicaría porque en este estudio la edad mayor de 60 años pareciera ser un factor protector para la IUE.

En el presente estudio se logró asociar significativamente a la obesidad con el desarrollo de IU, lo que concuerda con los estudios realizados 
en otros países ${ }^{4,18,19}$. También existe evidencia de que estrategias de reducción de peso se asocian a una reducción en los síntomas de $\mathrm{IU}^{34}$. Desde una mirada de salud pública la asociación establecida en este estudio entre obesidad e IU es un hallazgo de gran relevancia, dada la alta prevalencia de obesidad en la población chilena, donde $64,3 \%$ de las mujeres presenta exceso de peso y $30,7 \%$ de las mujeres son obesas ${ }^{35}$.

La paridad y el mecanismo de parto son factores de riesgo establecidos para IU, especialmente para IUE I, $2,4,7,23,34,36,37$. En este estudio no observamos una asociación significativa, pero si una tendencia mayor a presentar IU en las mujeres con al menos un embarazo. Una posible explicación para la falta de asociación es que la muestra de este estudio incluyó sólo una minoría de mujeres nulíparas, dificultando la capacidad de detectar el efecto de la nuliparidad en el desarrollo de IU. $\mathrm{Al}$ analizar los tipos de IU por separado se encontró que haber sido sometida a una episiotomía durante el parto aumenta significativamente la probabilidad de presentar IUE. Diversos estudios han encontrado una asociación similar ${ }^{24}$. Es importante resaltar la elevada tasa de episiotomía encontrada en este estudio (81,2\%). El impacto de este procedimiento sobre el desarrollo posterior de IUE es de importancia en Chile, ya que sería un factor modificable que podría reducir la incidencia de esta enfermedad en la población. El bajo porcentaje de mujeres sometidas a parto instrumental en este estudio dificultó la capacidad para detectar el posible efecto del mismo sobre el desarrollo de IU.

Por último, a pesar de que no se encontró una relación significativa entre presencia de IU y constipación, si se logró detectar que las mujeres constipadas presentan una mayor severidad de IU. Varios estudios ${ }^{33}$ fallaron en detectar un riesgo mayor en las mujeres constipadas de presentar IU. No existe literatura sobre la relación entre constipación y severidad de la IU, pero existe una opinión generalizada de que el pujo excesivo durante la defecación en pacientes con constipación crónica induce una neuropatía progresiva del piso pélvico que altera la función del sistema digestivo y urinario, lo que puede explicar un aumento en la severidad de los síntomas de incontinencia urinaria reportada en las pacientes de este estudio.

Una limitación del estudio es la selección de las mujeres, ya que se eligieron directamente en la unidad de la mujer del centro de salud. Sin embargo, y a pesar de que este estudio demuestra que la IU es un problema común, la tasa de consulta por esta enfermedad fue de sólo $10,7 \%$, la cual es mucho más baja que la reportada en países europeos $(24-40 \%)^{6}$; esto implica que una gran cantidad de mujeres chilenas que sufren este problema de salud nunca han hablado del mismo con un profesional de la salud, lo que se traduce en una baja tasa de tratamiento. Las razones por la que esto ocurre aún no están claramente establecidas. En un estudio, $\mathrm{Hsieh}^{38}$ encontró que las principales razones por las que las mujeres no buscaban tratamiento eran la vergüenza de hablar de la IU, el pensamiento de que esta es una condición normal, o el desconocimiento sobre cuál era el profesional apropiado para consultar por este problema. En el presente estudio el mayor porcentaje de consulta fue a las matronas de APS, por lo que los controles de salud de la mujer pueden considerarse un punto importante para la detección de esta patología en el sistema público. Se requiere investigación tanto epidemiológica como cualitativa en este campo para entender los factores que influencian a las mujeres chilenas a buscar ayuda o los limitantes que dificultan que lo hagan.

En la actualidad el manejo interdisciplinario de la IU, tanto preventivo como curativo, no es una prioridad a la hora de destinar recursos de salud. Ya que no se ha incorporado su manejo a ningún programa de salud existente, aquellas que la padecen deben enfrentar los costos económicos de forma independiente. Estos costos incluyen tanto los directos, asociados a tratamientos médicos o quirúrgicos, los costos derivados de insumos como apósitos y otros productos relacionados y también los costos indirectos, relacionados con la pérdida de capacidad laboral, y de oportunidades de participación social. Todos estos elementos deben ser estudiados en mayor profundidad, sin embargo, este estudio es un elemento contribuyente a detectar la importancia de este problema de salud, y de esta manera apoyar la toma de decisiones a la hora de distribuir los recursos, y elaborar programas de salud para las mujeres de Chile.

Agradecimientos: Al equipo de la unidad de la mujer del CESFAM Ultraestación de Chillán, por su gran apoyo y motivación para la elaboración de este estudio. 


\section{Referencias}

1. Melville J, Katon W, Delaney K, Newton K. Urinary Incontinence in US Women. A Population-Based Study. Arch Intern Med. 2005; 165 (5): 537-42.

2. Hannestad Y, Rortveit G, Sandvik H, Hunskaar S. A community-based epidemiological survey of female urinary incontinence: the Norwegian EPINCONT study. Epidemiology of Incontinence in the County of NordTrondelag. Journal of Clinical Epidemiology 2000; 53 (11): 1150-7.

3. Minassian V, Stewart W, Wood C. Urinary Incontinence in Women: Variation in Prevalence Estimates and Risk Factors. Obstetrics \& Gynecology 2008; 111 (2): 324-31.

4. Waetjen LE, Liao S, Johnson WO, Sampselle C, Sternfield B, Siobán D, Harlow SD, et al. Factors associated with prevalent and incident urinary incontinence in a cohort of midlife women: a longitudinal analysis of data: study of women's health across the nation. American Journal of Epidemiology 2007; 165 (3): 309-18.

5. Brown JS, Grady D, Ouslander JG, Herzog A, Varner $\mathrm{R}$, Posner SF. Prevalence of Urinary Incontinence and Associated Risk Factors in Postmenopausal Women. Obstetrics \& Gynecology 1999; 94 (1): 66-70.

6. Hunskaar S, Lose G, Sykes D, Voss S. The Prevalence of urinary incontinence in women in four European countries. BJU International 2004; 93 (3): 324-30.

7. Buckley BS, Lapitan MC. Prevalence of urinary incontinence in men, women, and children-current evidence: findings of the Fourth International Consultation on Incontinence. Urology 2010; 76 (2): 265-70.

8. Salazar A, Oyanedel P, Montiglio C, Campero JM, Caro $\mathrm{C}$, Monje B, et al. Prevalencia y factores de riesgo de la incontinencia de orina. Revista Chilena de Urología 2005; 7 (1): 55-8.

9. Harwardt T, Fuentes B, Venegas M, Leal C, Verdugo F, Benier P. Estudio de prevalencia de incontinencia urinaria en mujeres de Isla de Pascua. Revista Chilena de Urología 2004; 69 (1): 29-34.

10. Lose G. The burden of stress urinary incontinence. European Urology Supplements 2005; 4 (1): 5-10.

11. Coyne KS, Kvasz M, Ireland AM, Milsom I, Kopp ZS, Chapple CR. Urinary Incontinence and its Relationship to Mental Health and Health-Related Quality of Life in Men and Women in Sweden, the United Kingdom, and the United States. European Urology 2012; 61 (1): 88-95.

12. Knorst M, Resende T, Goldim JR. Clinical profile, quality of life and depressive symptoms of women with urinary incontinence attending a university hospital. Revista Brasileira de Fisioterapia 2011; 15 (2): 109-16.

13. Hu TW. Impact of urinary incontinence on health care costs. Journal of the American Geriatrics Society 1990; 38 (1): 292-9.

14. Subak L, Brown JS, Kraus SR, Brubaker L, Lin F. Richter $\mathrm{HE}$, et al. The "Costs" of Urinary Incontinence for Women. Obstetrics and Gynecology 2006; 107 (4): 908-16.

15. Stothers L, Friedman B. Risk Factors for the Development of Stress Urinary Incontinence in Women. Current Urology Reports 2011; 12: 363-9.

16. Luber KM, Boero S, Choe JY. The demographics of pelvic floor disorders: current observations and future projections. American Journal of Obstetrics and Gynecology 2001; 184 (7): 1496-503.

17. Peyrat L, Haillot O, Bruyere F, Boutin JF, Bertrand P, Lanson Y. Prevalence and risk factors of urinary incontinence in young and middle aged women. BJU International 2002; 89 (1): 61-6.

18. Subak L, Richter HE, Hunskaar S. Obesity and urinary incontinence: epidemiology and clinical research update. Journal of Urology 2009; 182 (6 Suppl): S2-7.

19. Hunskaar S. A systematic review of overweight and obesity as risk factors and targets for clinical intervention for urinary incontinence in women. Neurourology and Urodynamics 2008; 27 (8): 749-57.

20. Townsend MK, Danforth KN, Rosner B, Curhan GC, Resnick NM, Grodstein F. Body mass index, weight gain, and incident urinary incontinence in middle-aged women. Obstetrics and Gynecology 2007; 110 (2): 346-53.

21. Townsend MK, Curhan GC, Resnick NM, Grodstein F. BMI, waist circumference, and incident urinary incontinence in older women. Obesity 2008; 16 (4): 881-6.

22. Phelan S, Grodstein F, Brown JS. Clinical research in diabetes and urinary incontinence: what we know and need to know. Journal of Urology 2009; 182 (6) Supp 1: S14-7.

23. Rortveit G, Daltveit NK, Hannestad YS. Urinary incontinence after vaginal delivery or cesarean section. The New England Journal of Medicine 2003; 348: 900-7.

24. Foldspang A, Mommsen S, Djurhuus JC. Prevalent urinary incontinence as a correlate of pregnancy, vaginal childbirth, and obstetric techniques. American Journal of Public Health 1999; 89 (2): 209-12.

25. Subak L, Van Den Eeden S, Thom D. Creasman JM, Brown JS. Reproductive Risks for Incontinence Study at Kaiser Research Group. Urinary incontinence in women: Direct costs of routine care. American Journal of Obstetrics and Gynecology 2007; 197 (6): 596.e1-9.

26. Milsom I, Ekelund P, Molander U, Arvidsson L, Areskoug B. The influence of age, parity, oral contraception, hysterectomy and menopause on the prevalence of urinary incontinence in women. Journal of Urology 1993; 149 (6): 1459-62. 
Caracterización clínica de la incontinencia urinaria en mujeres - O. Rincón Ardila

27. Legendre G, Ringa V, Fauconnier A, Fritel X. Menopause, hormone treatment and urinary incontinence at midlife. Maturitas 2013; 74 (1): 26-30.

28. Altman D, Granath F, Cnattingius S, Falconer C. Hysterectomy and risk of stress-urinary-incontinence surgery: nationwide cohort study. Lancet 2007; 370: 1494-9.

29. Miller JJ, Botros SM, Baumont JM, Aschkenazi SO, Gamble T, Sand P, et al. Impact of hysterectomy on stress urinary incontinence: an identical twin study. American Journal of Obstetrics and Gynecology 2008; 198 (5): 565-70.

30. De Tayrac R, Chevalier N, Chauveaud-Lambling A, Gervaise A, Fernández H. Is vaginal hysterectomy a risk factor for urinary incontinence at long-term follow-up? European Journal of Obstetrics \& Gynecology and Reproductive Biology 2007; 130 (2): 258-61.

31. Sandvik H. A severity Index for Epidemiological Surveys of Female Urinary Incontinence: Comparison with 48Hour Pad-Weighing Tests. Neurourology and urodynamics 2000; 19 (2): 137-45.

32. Hunskaar S. Epidemiology and Natural History of Urinary Incontinence (UI). En: Abrams P, Cardozo L, Khoury S, Wein A. (Eds.). Incontinence. Plymbridge
Distributors Ltd., United Kingdom, 2002. p 165-202.

33. Minassian VA, Drutz HP, Al-Badr A. Urinary incontinence as a worldwide problem. International Journal of Gynecology and Obstetrics 2003; 82 (3): 327-38.

34. Subak LL, Wing R, Smith West D, Franklin F, Vittinghoff E, Creasman JM. Weight Loss to Treat Urinary Incontinence in Overweight and Obese Women. New England Journal of Medicine 2009; 360 (5): 481-90.

35. Ministerio de Salud. Primera Encuesta Nacional de Salud, En: http://epi.minsal.cl/epi/html/invest/ENS/ENS. html [Consultado el 7 de noviembre de 2012].

36. Rortveit G, Hannestad YS, Daltveit AK, Hunskaar S. Age-and type-dependent effects of parity on urinary incontinence: The Norwegian EPINCONT Study. Obstetrics \& Gynecology 2001; 98 (6): 1004-10.

37. Snooks SJ, Swash M, Mathers SE, Henry MM. Effect of vaginal delivery on the pelvic floor: a 5-year follow-up. British Journal of Surgery 1990; 77 (12): 1358-60.

38. Hsieh CH, Su TH, Chang ST, Lin SH, Lee MC, Lee MY. Prevalence of and attitude toward urinary incontinence in postmenopausal women. International Journal of Gynecology and Obstetrics 2008; 100 (2): 171-4. 